\title{
Scaling up and scaling down the production of galactaric acid from pectin using Trichoderma reesei
}

\author{
Toni Paasikallio, Anne Huuskonen and Marilyn G. Wiebe* (B)
}

\begin{abstract}
Background: Bioconversion of D-galacturonic acid to galactaric (mucic) acid has previously been carried out in small scale (50-1000 mL) cultures, which produce tens of grams of galactaric acid. To obtain larger amounts of biologically produced galactaric acid, the process needed to be scaled up using a readily available technical substrate. Food grade pectin was selected as a readily available source of D-galacturonic acid for conversion to galactaric acid.

Results: We demonstrated that the process using Trichoderma reesei QM6a $\triangle$ gar1 udh can be scaled up from $1 \mathrm{~L}$ to 10 and $250 \mathrm{~L}$, replacing pure D-galacturonic acid with commercially available pectin. T. reesei produced $18 \mathrm{~g} \mathrm{~L}^{-1} \mathrm{galac}-$ taric acid from food-grade pectin (yield $1.00 \mathrm{~g}$ [g D-galacturonate consumed] ${ }^{-1}$ ) when grown at $1 \mathrm{~L} \mathrm{scale,} 21 \mathrm{~g} \mathrm{~L}^{-1}$ galactaric acid (yield $1.11 \mathrm{~g}$ [g D-galacturonate consumed $]^{-1}$ ) when grown at $10 \mathrm{~L} \mathrm{scale} \mathrm{and} 14 \mathrm{~g} \mathrm{~L}^{-1}$ galactaric acid (yield $0.77 \mathrm{~g}$ [g D-galacturonate consumed $]^{-1}$ ) when grown at $250 \mathrm{~L}$ scale. Initial production rates were similar to those observed in $500 \mathrm{~mL}$ cultures with pure D-galacturonate as substrate. Approximately $2.8 \mathrm{~kg}$ galactaric acid was precipitated from the $250 \mathrm{~L}$ culture, representing a recovery of $77 \%$ of the galactaric acid in the supernatant. In addition to scaling up, we also demonstrated that the process could be scaled down to $4 \mathrm{~mL}$ for screening of production strains in 24-well plate format. Production of galactaric acid from pectin was assessed for three strains expressing uronate dehydrogenase under alternative promoters and up to $11 \mathrm{~g} \mathrm{~L}^{-1}$ galactaric acid were produced in the batch process.

Conclusions: The process of producing galactaric acid by bioconversion with T. reesei was demonstrated to be equally efficient using pectin as it was with D-galacturonic acid. The 24-well plate batch process will be useful screening new constructs, but cannot replace process optimisation in bioreactors. Scaling up to $250 \mathrm{~L}$ demonstrated good reproducibility with the smaller scale but there was a loss in yield at $250 \mathrm{~L}$ which indicated that total biomass extraction and more efficient DSP would both be needed for a large scale process.
\end{abstract}

Keywords: Galactaric acid, Mucic acid, D-Galacturonic acid, Pectin, Trichoderma reesei, Scale-up, Scale-down

\section{Background}

There is considerable interest in replacing chemicals derived from petroleum with bio-derived chemicals, i.e. chemicals obtained by bioconversion and/or chemical conversion from renewable biological resources, primarily plants. Both galactaric and glucaric acids have been identified as substrates for chemical conversion to adipic

*Correspondence: Marilyn.Wiebe@vtt.fi

VTT Technical Research Centre of Finland Ltd., P.O. Box 1000, 02044 Espoo, Finland acid, furandicarboxylic acid (which is being developed as a substitute for terephthalic acid) and other platform chemicals, including anhydrides, diesters and diallyls which can be used in the synthesis of higher value products [1-3]. Both compounds can be prepared by nitric acid oxidation of the corresponding monosaccharide (D-glucose or D-galactose) [4]. Although biotechnological conversions may be preferable to nitric acid oxidation, biotechnological conversion of D-glucose to glucaric acid continues to be challenging [5]. However, galactaric acid has been produced from D-galacturonic acid using 
genetically modified Escherichia coli and Trichoderma reese $i$ at concentrations of 10 [6] to 20 [7] $\mathrm{g} \mathrm{L}^{-1}$, which are high enough concentrations for precipitation from the culture broth, making biotechnologically derived galactaric acid available for assessment in further chemical conversions [6]. The genetically modified $E$. coli or $T$. reesei convert $\mathrm{D}$-galacturonic acid to galactaric acid by the action of a uronate dehydrogenase (UDH), which is expressed in a strain in which normal D-galacturonic acid metabolism has been disrupted $[6,8]$. In the case of $T$. reesei, deletion of the gene encoding NADPH-dependent D-galacturonate reductase ( $\operatorname{ar} 1$ ), the first step in the fungal pathway for D-galacturonic acid metabolism, is sufficient to disrupt the pathway and expression of the $A$. tumefaciens $u d h$ gene results in a strain which produces galactaric acid [8].

Although chemically produced galactaric acid is commercially available, biotechnologically produced galactaric acid will contain different impurities and the effect of these on specific chemical reactions needs to be assessed to provide feedback on the purity requirements and consequent implications for downstream processing (DSP) in the biotechnological process. For this reason it may be necessary to produce substantial quantities of galactaric acid using biotechnology, even though the process is not ready for commercialisation.

The substrate for biotechnological production of galactaric acid is D-galacturonic acid, which can be obtained by hydrolysis of pectin. Zhang et al. [6] demonstrated that galactaric acid could be produced from enzymatically hydrolysed sugar beet pulp, but noted that the yield of galactaric acid on sugar beet pulp was relatively low (0.14 g galactaric acid per g sugar beet pulp), reflecting the high concentration of other compounds in the pulp, particularly $\mathrm{D}$-glucose and $\mathrm{L}$-arabinose. D-Galacturonic acid is currently available only at high prices (more than $€ 3000$ per $\mathrm{kg}$ ) and is thus not suitable for producing kilogram amounts of galactaric acid for chemical testing. However, food grade pectins are commercially available at prices in the range of $€ 10-100$ per $\mathrm{kg}$, making them a reasonable source for preparation of large amounts of galactaric acid. It should be noted that food grade pectin may be diluted with additives such as sucrose or glucose. Having received a request to provide a $2 \mathrm{~kg}$ sample of biotechnologically produced galactaric acid for use in chemical reactions, we decided to use pectin as the source of $\mathrm{D}$-galacturonic acid in scaling up the process of galactaric acid production with $T$. reesei $\Delta$ gar1 $u d h$. An industrial process would be expected to use sugar beet pulp, citrus waste and other pectin-rich waste streams or crude extractions of pectin from these sources, which would not compete with the food use of current pectin production, but these were not readily available at the scale needed. $T$. reesei $\Delta$ gar1 $u d h$ was used in scaling up the process since it has already been demonstrated to produce up to $20 \mathrm{~g} \mathrm{~L}^{-1}$ galactaric acid from $\mathrm{D}$-galacturonic acid [7]. Since T. reesei does not hydrolyse pectin [9] and to facilitate sterilisation of viscous pectin solutions, enzymatic pre-hydrolysis of the pectin was necessary. An alternative approach would be to develop a consolidated process using a production strain such as Aspergillus niger, which produces native pectinases. However, $A$. niger can metabolise galactaric acid [8] and a strain in which this pathway has been disrupted has only recently become available [10]. A production process with $A$. niger has not yet been developed.

In addition to scaling up the process of producing galactaric acid to provide galactaric acid for chemical reactions, we were also interested in providing a scaled down process, which would enable the screening of new strains [11]. Galactaric acid production by T. reesei has previously been demonstrated in flask cultures [8], but at much lower concentrations than can be obtained in bioreactors [7]. Running and Bansal [12] demonstrated that 24-well plates can have as good or better oxygen transfer as shaken flasks, depending on the shaking regime applied, and 24-well plates are increasingly being used for the cultivation of filamentous fungi [13-15]. It is therefore useful to assess whether galactaric acid production by $T$. reesei could be scaled down for production in 24-well plates using pectin as substrate.

In this paper we describe the production of galactaric acid by $T$. reesei VTT D-161646 from enzyme hydrolysed pectin at 1,10 and $250 \mathrm{~L}$ scales. We also demonstrate that the process can be scaled down to $4 \mathrm{~mL}$ for strain screening, for example in considering the effectiveness of alternative promoters for expression of the uronate dehydrogenase gene, as shown here.

\section{Results}

Scaling down production of galactaric acid in 24-well plates

Three transformants of $T$. reesei were generated in which the uronate dehydrogenase $(u d h)$ gene was expressed under different promoters and these were cultivated in 24-well plates to assess the suitability of the 24-well plate format for galactaric acid production. All transformants which contained the $u d h$ gene produced galactaric acid in the 24-well plates. T. reesei VTT D-161646 produced $8.8 \mathrm{~g} \mathrm{~L}^{-1}$ galactaric acid from $10.7 \mathrm{~g}$ D-galacturonic acid in 6 days and $10.5 \mathrm{~g} \mathrm{~L}^{-1}$ from $20 \mathrm{~g} \mathrm{~L}^{-1}$ pectin (Sigma), hydrolysed to give $11.6 \mathrm{~g} \mathrm{~L}^{-1}$ D-galacturonic acid, demonstrating that galactaric acid could be produced in the 24-well plates (Fig. 1; Table 1). No galactaric acid was produced by M122, the control strain lacking $u d h$, nor in wells which had not been 

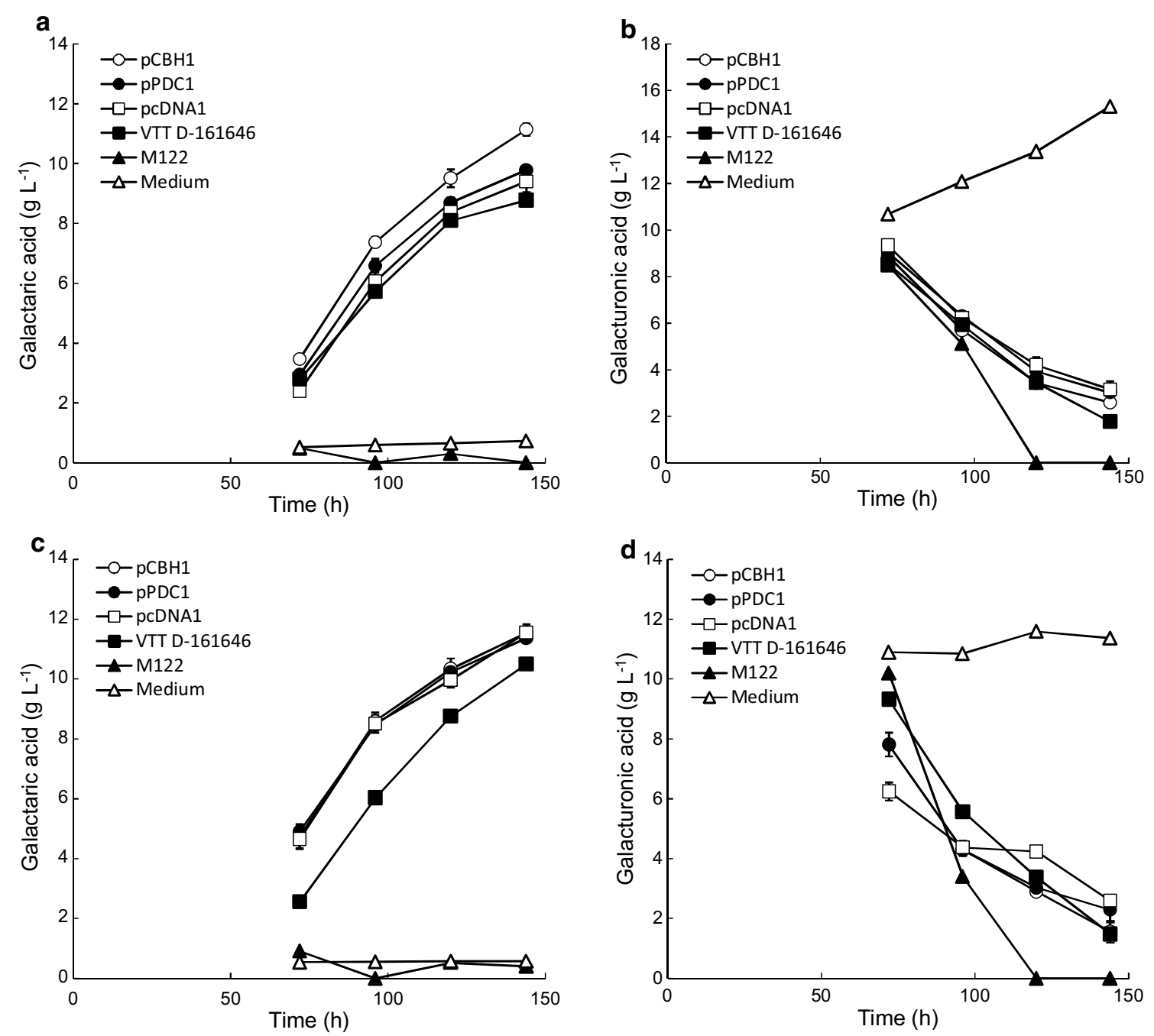

Fig. 1 Galactaric acid production and D-galacturonic acid consumption in 24-well plates. T. reesei $\triangle$ gar1 udh strains, in which the udh gene was under control of the CBH1, PDC1, CDNA1 or GPDA (VTT D-161646) promoters, and M122, which contains gar1 and does not contain udh, were grown in $4 \mathrm{~mL}$ medium containing pure D-galacturonate $(\mathbf{a}, \mathbf{b})$ or hydrolysed pectin $(\mathbf{c}, \mathbf{d})$ as substrate in 24-well plates. All values have been adjusted for evaporation, except those for the medium in wells with pure $\mathrm{D}$-galacturonic acid. Values for $\mathrm{pCBH}$, pPDC1 and pcDNA1 represent mean \pm sem for $\mathrm{n}=3$. Where not seen, error bars were smaller than the symbols

inoculated (Fig. 1). The increase in concentration of D-galacturonic acid (Fig. 1b) and lactose in the uninoculated wells provided evidence of the extent of evaporation in the wells and this data was used to calculate the actual concentrations of galactaric acid produced in other wells. Hydrolysis of the pectin was not complete within $72 \mathrm{~h}$, so changes in the concentrations of $\mathrm{D}$-galacturonic acid and lactose in the well with medium containing D-galacturonate were used to adjust for evaporation in the pectin containing wells.

Each of the new transformants produced similar amounts of galactaric acid to VTT D-161646 and to each other (Fig. 1; Table 1). The strain expressing $u d h$ under control of the CBH1 (cellobiohydrolase I) promoter produced significantly more $(\mathrm{p}<0.05)$ galactaric acid $\left(11.2 \pm 0.2 \mathrm{~g} \mathrm{~L}^{-1}\right)$ from $\mathrm{D}$-galacturonic acid than the other three strains, but there were no significant differences $(\mathrm{p}>0.05)$ in the amounts produced from Sigma pectin (Fig. 1). The yield of galactaric acid on D-galacturonic acid was between 0.87 and $1.00 \mathrm{~g} \mathrm{~g}^{-1}$ in the 24 -well plates when D-galacturonic acid was used as the substrate, and between 0.76 and $0.90 \mathrm{~g} \mathrm{~g}^{-1}$ when pectin was used as substrate (Table 1). Production rates between 72 and $120 \mathrm{~h}$ were $0.11-0.13 \mathrm{~g} \mathrm{~L}^{-1} \mathrm{~h}^{-1}$ with either pure D-galacturonic acid or with pectin as the source of D-galacturonic acid (Table 1). 
Table 1 Galactaric acid production by T. reesei expressing udh under promoters pCBH1, pCDNA1, pPDC1 or pGPDA

\begin{tabular}{|c|c|c|c|c|}
\hline Strain & $\mathrm{pCBH} 1$ & pcDNA1 & $\mathrm{p} P D C 1$ & $\mathrm{pGPDA} A^{\mathrm{a}}$ \\
\hline \multicolumn{5}{|l|}{ 24-well plates_-D-galacturonate } \\
\hline Galactaric acid $\left(\mathrm{g} \mathrm{L}^{-1}\right)$ & $11.1 \pm 0.2$ & $9.4 \pm 0.4$ & $9.8 \pm 0.1$ & 8.8 \\
\hline Galactaric acid production rate $(72-120 \mathrm{~h})\left(\mathrm{g} \mathrm{l}^{-1} \mathrm{~h}^{-1}\right)$ & $0.13 \pm 0.01$ & $0.12 \pm 0.00$ & $0.12 \pm 0.00$ & 0.11 \\
\hline Yield galactaric/D-galacturonic $\left(\mathrm{g} \mathrm{g}^{-1}\right)$ & $0.95 \pm 0.02$ & $0.87 \pm 0.02$ & $0.89 \pm 0.00$ & 0.89 \\
\hline \multicolumn{5}{|l|}{ 24-well plates-pectin } \\
\hline Galactaric acid $\left(\mathrm{g} \mathrm{L}^{-1}\right)$ & $11.6 \pm 0.2$ & $11.5 \pm 0.3$ & $11.4 \pm 0.1$ & 10.5 \\
\hline Galactaric acid production rate $(72-120 \mathrm{~h})\left(\mathrm{g} \mathrm{L}^{-1} \mathrm{~h}^{-1}\right)$ & $0.12 \pm 0.04$ & $0.11 \pm 0.00$ & $0.11 \pm 0.00$ & 0.13 \\
\hline Yield galactaric/D-galacturonic $\left(\mathrm{g} \mathrm{g}^{-1}\right)$ & $0.84 \pm 0.03$ & $0.90 \pm 0.03$ & $0.89 \pm 0.03$ & 0.76 \\
\hline \multicolumn{5}{|l|}{$0.5 \mathrm{~L}$ bioreactors—D-galacturonate } \\
\hline Galactaric acid $\left(\mathrm{g} \mathrm{L}^{-1}\right)$ & 15.7 & 13.6 & 16.0 & 19.7 \\
\hline Galactaric acid production rate $(0-72 \mathrm{~h})\left(\mathrm{g} \mathrm{L}^{-1} \mathrm{~h}^{-1}\right)$ & 0.22 & 0.21 & 0.09 & 0.19 \\
\hline Yield galactaric/D-galacturonic $\left(\mathrm{g} \mathrm{g}^{-1}\right)$ & 0.90 & 0.82 & 1.11 & 0.85 \\
\hline Maximum biomass $\left(\mathrm{g} \mathrm{L}^{-1}\right)$ & $9.5 \pm 0.2$ & $9.0 \pm 0.3$ & $6.5 \pm 0.5$ & $7.9 \pm 0.3$ \\
\hline
\end{tabular}

Galactaric acid production (titre, rate of production and yield on D-galacturonic acid) in 24-well plates and in $0.5 \mathrm{~L}$ bioreactors using either D-galacturonic acid or pectin (Sigma) as substrate, with lactose as co-substrate. Maximum biomass in bioreactors was observed at 50 (pCBH1, pcDNA1, pGPDA) or 72 ( $\mathrm{pPDC1}$ ) h after which time biomass decreases (see also Fig. 3). Values are mean \pm sem for three replicates

a Strain VTT D-161646

In lactose-D-galacturonic acid fed-batch cultures $(0.5 \mathrm{~L}$, using the optimised process described by [7]) transformants expressing $u d h$ under the gpdA (glyceraldehyde3-phosphate dehydrogenase, strain VTT D-161646), $C B H 1, c D N A 1$ (unidentified hypothetical protein), and $P D C 1$ (pyruvate decarboxylase) promoters produced 20, 16,14 and $16 \mathrm{~g} \mathrm{~L}^{-1}$ galactaric acid, respectively (Table 1 ). Yields were $0.85,0.90,0.82$ and $1.11 \mathrm{~g}$ galactaric acid [g D-galacturonate consumed] ${ }^{-1}$, respectively. However, the strain producing galactaric acid under the $p d c 1$ promoter grew slower than the other strains, producing less biomass, and the initial production rate (between 0 and $72 \mathrm{~h})$ of D-galactaric acid $\left(0.09 \mathrm{~g} \mathrm{~L}^{-1} \mathrm{~h}^{-1}\right)$ was slower than that of the other three strains $\left(0.19-0.20 \mathrm{~g} \mathrm{~L}^{-1} \mathrm{~h}^{-1}\right.$, Table 1).

\section{Production of galactaric acid from pectin at 1, 10 and $250 \mathrm{~L}$ scale}

Strain VTT D-161646 was used in the scaling up experiments since production conditions have been developed specifically for this strain [7] and there was no improvement in galactaric acid production with the new strains under these conditions. In order to scale up production of galactaric acid, D-galacturonic acid was replaced with pectin, using either Sigma or Meridianstar pectin (Table 2). A process scheme for the production of galactaric acid from pectin is shown in Fig. 2. The $10 \mathrm{~L}$ culture was carried out using Sigma pectin while waiting for the Meridianstar pectin to arrive. Because of the high D-glucose content in the Meridianstar pectin it was necessary to adjust the process at $1 \mathrm{~L}$ scale to assess the extent to which the high D-glucose content would repress
Table 2 Carbohydrate composition (\% dry matter) of pectin

\begin{tabular}{lll}
\hline Pectin & Sigma P1935 & $\begin{array}{l}\text { Meridianstar } \\
\text { rapid set }\end{array}$ \\
\hline D-Galacturonate & 65.6 & 43.0 \\
D-Glucose & 1.7 & 35.5 \\
L-Arabinose & 1.2 & 1.5 \\
D-Galactose + D-xylose & 11 & 4 \\
\hline
\end{tabular}

Pectin was hydrolysed by addition of $0.5-1.0 \mathrm{~mL} \mathrm{~L}^{-1}$ Pectinex Ultra with $0.1 \mathrm{~mL} \mathrm{~L}^{-1}$ Pectinex Smash and incubation at $40^{\circ} \mathrm{C}$. D-Galactose and D-xylose were not separated on the HPLC column used

galactaric acid production. Therefore both 1 and $250 \mathrm{~L}$ cultures were carried out using Meridianstar pectin.

When Sigma pectin replaced D-galacturonic acid in the $T$. reese $i$ galactaric acid production process, $21 \mathrm{~g} \mathrm{~L}^{-1}$ galactaric acid was produced with a yield of $1.11 \mathrm{~g}$ galactaric acid [g D-galacturonic acid $]^{-1}$, assuming that the pectin had been fully hydrolysed. The yield of galactaric acid on pectin was $0.73 \mathrm{~g} \mathrm{~g}^{-1}$. The production rate during the first $140 \mathrm{~h}\left(0.14 \mathrm{~g} \mathrm{~L}^{-1} \mathrm{~h}^{-1}\right)$ was comparable to that observed previously with pure $\mathrm{D}$-galacturonic acid during the same time interval $\left(0.15 \mathrm{~g} \mathrm{~L}^{-1} \mathrm{~h}^{-1}\right.$; Fig. 3) [7] and the process generally showed good reproducibility with the $500 \mathrm{~mL}$ scale production from D-galacturonic acid. No accumulation of D-galacturonic acid or other carbohydrates (lactose, glucose, galactose or arabinose) was observed during the feeding phase. Biomass production on pectin with lactose was a bit higher than on D-galacturonic acid with lactose, even though the lactose concentration had been reduced to take into account the 

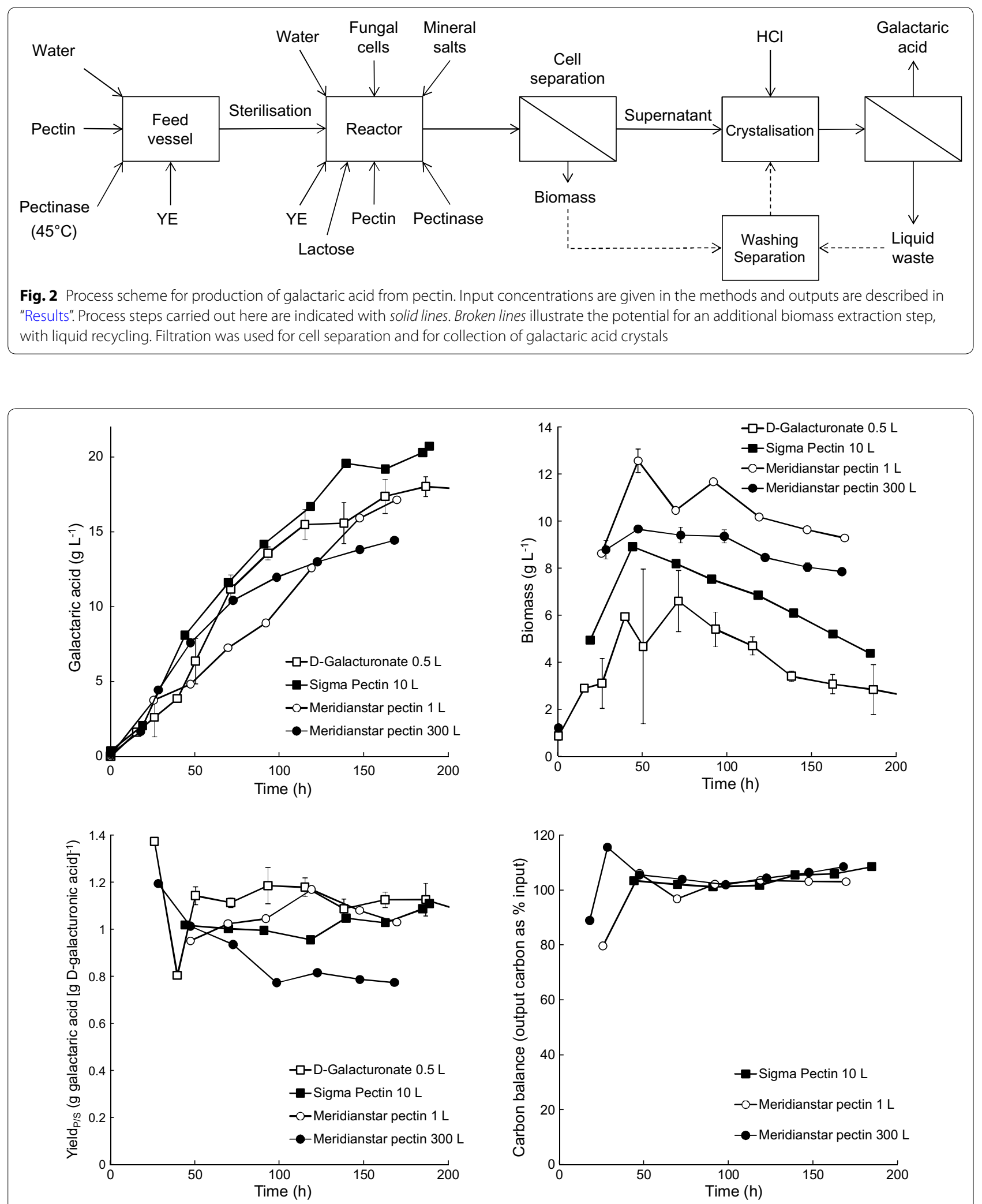

Fig. 3 Galactaric acid and biomass production at 0.5, 1, 10 and $250 \mathrm{~L}$ scale. T. reesei $\Delta$ gar 1 udh VTT D-161646 was provided D-galacturonic acid $(0.5 \mathrm{~L}$, [7]) or hydrolysed pectin $(1,10$ and $250 \mathrm{~L})$ as substrate with lactose as co-substrate. The approximate yield of galactaric acid on D-galacturonic acid and the overall carbon balance are also shown. Values for production from D-galacturonic acid at $0.5 \mathrm{~L}$ scale are mean \pm sem for 4 independent cultivations. Error bars for biomass measurements at 1, 10 and $300 \mathrm{~L}$ scale are \pm sem for duplicate measurements 
carbohydrates introduced with the pectin (Fig. 3). D-Glucose remained limiting throughout the feeding phase.

The culture broth was collected at the end of the cultivation and the biomass separated by filtration, generating $8.8 \mathrm{~L}$ clear liquid and $282 \mathrm{~g}$ wet biomass. Precipitating and collecting the crystals of galactaric acid resulted in $154 \mathrm{~g}$ galactaric acid, representing $85 \%$ of the galactaric acid produced (Table 3 ).

Using Meridianstar Rapid Set pectin, $19 \mathrm{~g} \mathrm{~L}^{-1}$ galactaric acid was produced with a yield of $1.00 \mathrm{~g}$ galactaric acid [g D-galacturonic acid $]^{-1}$, assuming complete hydrolysis of the pectin (Fig. 3). The yield of galactaric acid on Meridianstar pectin was $0.41 \mathrm{~g} \mathrm{~g}^{-1}$. Feeding was not started until all D-glucose and lactose had been consumed, but this resulted in D-galacturonic acid limitation during the batch phase and reduced the initial galactaric acid production rate. The initial production rate $(0-70 \mathrm{~h})$ was only $0.10 \mathrm{~g} \mathrm{~L}^{-1} \mathrm{~h}^{-1}$, but during the feeding phase $(90-150 \mathrm{~h})$ it increased to $0.13 \mathrm{~g} \mathrm{~L}^{-1} \mathrm{~h}^{-1}$. Although there was no accumulation of $\mathrm{D}$-glucose during the feeding phase, there was initial accumulation of D-galacturonic acid (up to $4.3 \mathrm{~g} \mathrm{~L}^{-1}$ ) in the culture supernatant and the feed rate was reduced to allow more complete conversion to D-galactaric acid. More biomass was produced when T. reesei VTT D-161646 was grown on Meridianstar pectin than on Sigma pectin and the biomass concentration was maintained during the feeding phase (Fig. 3). Approximately $89 \%$ of the galactaric acid was recovered by precipitation in acid (Table 3 ).
Based on the results at the $1 \mathrm{~L}$ scale, T. reesei VTT D-161646 was grown in a $300 \mathrm{~L}$ bioreactor and $256 \mathrm{~L}$ of $14 \mathrm{~g} \mathrm{~L}^{-1}$ galactaric acid was produced. The initial $(0-73 \mathrm{~h})$ production rate of $0.15 \mathrm{~g} \mathrm{~L}^{-1} \mathrm{~h}^{-1}$ was only sustained for $72 \mathrm{~h}$, after which time both the rate of galactaric acid production and the yield of galactaric acid on D-galacturonic acid decreased. The final yield was $0.77 \mathrm{~g}$ galactaric acid [g D-galacturonic acid] ${ }^{-1}$ or $0.32 \mathrm{~g}$ [g pectin $]^{-1}$ (Fig. 3). Crystals were observed among the mycelia under the microscope and were also present in the foam which had been produced in the bioreactor. After precipitation, collection and drying, $2.81 \mathrm{~kg}$ galactaric acid was obtained, or $73 \%$ of the amount present in the supernatant (Table 3). Biomass was not extracted.

\section{Discussion}

The process of producing galactaric acid using T. reesei was scalable both up and down, with little loss in productivity, even with the substitution of pectin for D-galacturonic acid. Pectin was found to be a suitable substrate for galactaric acid production, although for T. reesei this required the addition of commercial pectinases. Addition of pectinases also facilitated sterilisation of the pectin feed, so even though $T$. reesei could be engineered to express a full range of pectinases to reduce the cost of enzyme addition, a fully consolidated process may not be desirable. The only other adjustment required for the use of pectin was a reduction in the amount of added co-substrate, since cosubstrates were provided with the pectin, particularly in

Table 3 Process parameters for galactaric acid production at $0.5,1,10$ and $250 \mathrm{~L}$

\begin{tabular}{|c|c|c|c|c|}
\hline & D-Galacturonic acid ${ }^{\mathrm{a}}$ & Meridianstar pectin & Sigma pectin & Meridianstar pectin \\
\hline Reactor volume $(\mathrm{L})$ & 0.5 & 1 & 10 & 250 \\
\hline Process time (h) & 242 & 215 & 185 & 168 \\
\hline \multicolumn{5}{|l|}{ Inputs } \\
\hline Lactose $\left(\mathrm{g} \mathrm{L}^{-1}\right)$ & 28 & 10 & 14 & 9 \\
\hline Galacturonic acid $\left(\mathrm{g} \mathrm{L}^{-1}\right)$ & 21 & & & \\
\hline Pectin $\left(\mathrm{g} \mathrm{L}^{-1}\right)$ & & 48.4 & 29.2 & 45.9 \\
\hline Pectinase(s) $\left(\mathrm{mL} \mathrm{L}^{-1}\right)$ & & 1 & 1 & 2 \\
\hline Fungal cells ( $\left.g \mathrm{~L}^{-1}\right)$ & 1 & 1 & 1 & 1 \\
\hline Air (vvm) & 1.4 & 0.5 & 0.5 & 0.5 \\
\hline \multicolumn{5}{|l|}{ Output } \\
\hline Galactaric acid $\left(\mathrm{g} \mathrm{L}^{-1}\right)$ & $21 \pm 2$ & 18 & 21 & 15 \\
\hline Yield (g [g galacturonate $]^{-1}$ ) & $1.0 \pm 0.2$ & 1.0 & 1.1 & 0.8 \\
\hline Yield (g [g pectin] $]^{-1}$ ) & & 0.41 & 0.73 & 0.32 \\
\hline Biomass $\left(\mathrm{g} \mathrm{L}^{-1}\right)^{\mathrm{b}}$ & $4 \pm 1$ & $9 \pm 0.1$ & $4 \pm 0.1$ & $8 \pm 0.2$ \\
\hline Product recovery $(\mathrm{kg})$ & & 0.014 & 0.15 & 2.8 \\
\hline Product recovery (\%) & $68 \pm 7$ & 89 & 85 & 73 \\
\hline
\end{tabular}

a Data from [7]. Values are mean \pm sem for four cultivations

b Biomass measurements are mean \pm sem for $2-3$ replicates, except for $0.5 \mathrm{~L}$ cultivations (mean \pm sem for 4 cultivations), at end of cultivation 
the case of the food-grade pectin, which contained a high concentration of D-glucose. Earlier results had indicated that D-glucose-limited fed-batch culture was less effective than lactose-limited fed-batch culture for galactaric acid production with $T$. reesei VTT D-161646 [7], but this did not appear to be the case when D-glucose was supplied with hydrolysed pectin in the feed.

Pectin was also used in the 24-well plates, with no apparent problems with mixing. Evaporation could be a problem at this scale, however, as seen in the increasing concentration of substrates in uninoculated wells, and needed to be taken into account. Production from hydrolysed pectin was comparable to that observed when pure D-galacturonic acid was provided. Production of galactaric acid was much higher in these $4 \mathrm{~mL}$ batch cultures than has previously been reported for flask cultures with filamentous fungi (around $4 \mathrm{~g} \mathrm{~L}^{-1} ;[8,10]$ ). The medium in the 24-well plates contained spent grain extract, which was expected to induce the CBH1 promoter [16], but not necessarily other promoters. Spent grain extract also provides organic nitrogen, resulting in a richer medium, which may contribute to the good conversion of D-galacturonic acid into galactaric acid. Kuivanen et al. [10] recently found that providing yeast extract and peptone to a A. niger galactaric acid producing strain, resulted in improved galactaric acid production, although the amounts were still low.

Production of galactaric acid by three new strains was compared in the 24-well plates, but production of all strains was found to be similar. This suggested that the amount of uronate dehydrogenase produced under the control of each of the promoters tested $(\mathrm{p} C B H 1$, $\mathrm{p} c D N A 1, \mathrm{p} P D C 1$ and the original $\mathrm{p} G P D A$ ) was adequate to catalyse the conversion of $\mathrm{D}$-galacturonic acid to galactaric acid at the rate at which it was taken up under these conditions. The effectiveness of these strains in producing galactaric acid was confirmed in $0.5 \mathrm{~L}$ bioreactors, however, the bioreactor cultures also revealed a slow growth (and production) phenotype for the strain expressing $u d h$ under the $P D C 1$ promoter, which was not observed in the microtiter plates. The $P D C$ promoter is expected to be particularly well induced when D-glucose is the main carbon source, and conversion of D-galacturonic to galactaric acid may be better with D-glucose as co-substrate [17], rather than lactose, as used here. The provision of better aeration and substrate-limited feeding in the bioreactors may also contribute to the phenotype.

The yield of galactaric acid on D-galacturonate was close to the theoretical yield of $1.08 \mathrm{~g} \mathrm{~g}^{-1}$ in the 1 and $10 \mathrm{~L}$ cultures, but in the $250 \mathrm{~L}$ culture the yield decreased to $0.8 \mathrm{~g}$ galactaric acid [g D-galacturonate consumed] ${ }^{-1}$ after $72 \mathrm{~h}$. The carbon balance (Fig. 3) indicated that all of the input carbon was accounted for in the output.
This suggests that galactaric acid crystals may have been included with inadequately washed biomass in the dry weight determination, or that some D-galacturonate contributed to $\mathrm{CO}_{2}$ production through an unknown pathway. Up to around $7 \%$ galactaric acid was expected to be removed with the biomass, but is expected to be washed off during rinsing for the dry weight determination. However, when harvesting the $250 \mathrm{~L}$ culture we observed a decrease in galactaric acid concentration during the filtration process, which indicated that galactaric acid crystals were trapped as more biomass accumulated in the filter. The concentration of galactaric acid was determined after filtration. Large scale extraction of the biomass was not possible at this time, but it is clear that this would be needed to obtain the maximum galactaric acid output from a scaled up process.

In spite of the low yield at the $250 \mathrm{~L}$ scale, around $3.8 \mathrm{~kg}$ galactaric acid was produced and $2.8 \mathrm{~kg}$ was recovered by acid precipitation. Poor mixing and inadequate cooling in the vessels used for the crystallisation step will have contributed to the recovery of only $73 \%$ of the galactaric acid. This was adequate for supplying material for further chemical conversions, but highlights the need for further DSP development.

\section{Conclusions}

The process of producing galactaric acid by bioconversion with $T$. reesei was demonstrated to be equally efficient using hydrolysed pectin as D-galacturonic acid as the substrate, and D-glucose present with the pectin did not inhibit the process. Scaling up to $250 \mathrm{~L}$ demonstrated good reproducibility with the smaller scale but there was a loss in yield at $250 \mathrm{~L}$ which indicated that both total biomass extraction and more efficient DSP would be needed for a large scale process. It would also be necessary to obtain higher production titres and production rates before a biotechnological process for producing galactaric acid would be considered for commercial production. However, the low solubility of galactaric acid [7] made it feasible to produce kilogram amounts of galactaric acid with the currently available strains so that assessment on purity requirements and further chemical transformations can proceed in parallel with further strain engineering. The 24-well plate batch process will be useful in screening new constructs, but cannot replace process optimisation in bioreactors.

\section{Methods}

Strains

Trichoderma reesei QM6a (VTT D-071262T, ATCC13631), M122 (RutC-30 mus53-), M1123 (M122 pyr4-) and VTT D-161646 were obtained from VTT's strain collections. Spore suspensions were prepared 
by cultivating the fungus on potato-dextrose agar (BD, Sparks, Maryland, USA) for 5-7 days, after which the spores were harvested, suspended in a buffer containing $0.8 \% \mathrm{NaCl}, 0.025 \%$ Tween- 20 and $20 \%$ glycerol, filtered through cotton and stored at $-80{ }^{\circ} \mathrm{C}$. Saccharomyces cerevisiae strain H3488 (FY834, obtained from Jay C. Dunlap) was used to clone expression construct plasmids. Escherichia coli TOP10 (Invitrogen) was used for propagation of the plasmids.

\section{Expression vectors}

Expression plasmids contained the $5^{\prime}$ and $3^{\prime}$ flanking regions of T. reesei D-galacturonic acid reductase (garl, [18]), T. reesei promoter (cbh1, cDNA1 or $p d c 1)$, Agrobacterium tumefasciens UDH gene, Aspergillus trpC terminator, T. reesei pyr4 selection marker with a loop-out fragment for marker removal, and the pRS426 plasmid as vector backbone.

Plasmid pRS426 (ATCC77107), used in cloning expression construct plasmids in yeast, was obtained from Jay C. Dunlap. For cloning, pRS426 was digested with restriction enzymes EcoRI and XhoI. A plasmid containing A. tumefasciens D-galacturonic acid dehydrogenase ( $u d h$ gene, GenBank no. BK006462.1) codon optimised for Aspergillus niger, [8] was digested with $\mathrm{NcoI}$ and SacII to release the $u d h$ gene from the vector backbone. The $5^{\prime}$ and $3^{\prime}$ flank (1572 and $1500 \mathrm{bp}$ ) fragments of garl, the three promoters (cbh1, cDNA1 and $p d c 1)$ and 285 bp of the garl $3^{\prime}$ flank as a direct repeat for looping out the selection marker were obtained by PCR using strain QM6a as template. The Aspergillus $\operatorname{trp} C$ terminator (773 bp) was obtained by PCR from a plasmid containing the element. PCR amplification was performed with a KAPA HiFi HotStart ReadyMix PCR kit (KAPABiosystems) using the primers listed in Table 4. The selection marker pyr4 was obtained from an existing plasmid with NotI digestion. All PCRs and digestions were separated with agarose gel electrophoresis and correct fragments were extracted with a gel extraction kit (Qiagen).

The appropriate purified DNA fragments were transformed to S. cerevisiae FY834 using the method described in Gietz and Woods [19]. The plasmids obtained through homologous recombination reactions were isolated from $S$. cerevisiae, amplified in $E$. coli and checked by restriction enzyme digestions and sequencing.

\section{Strain generation}

Expression plasmids $(10 \mu \mathrm{g})$ were digested with MssI, resulting in approximately $6 \mu \mathrm{g}$ of released expression cassette from each. To generate $3^{\prime}$ single stranded overhangs and thus to improve transformation efficiency, digestion mixtures were further treated with T7 exonuclease [20] and used without further purification.

Strain M1123 was transformed with each of the three expression constructs and positive transformants were selected on minimal medium as described previously [21, 22]. Transformants were sub-cultured onto agar-solidified minimal medium containing $1 \mathrm{~mL} \mathrm{~L}^{-1}$ Triton X-100. The isolates were screened by PCR to verify correct integration at the garl locus and a few correctly integrated transformants were purified as single spore isolates. Deletion of gar1 was verified by PCR.

\section{Media}

The low phosphate medium described by [7] was used for 24-well plate, flask and bioreactor cultivations, with D-galacturonic acid, pectin and lactose provided as

Table 4 Primers used to generate fragments for cloning by PCR amplification

\begin{tabular}{|c|c|c|c|}
\hline Product & Primer & Sequence & $\begin{array}{l}\text { Product } \\
\text { size (bp) }\end{array}$ \\
\hline \multirow[t]{2}{*}{ gar1 5'flank (tre22004) } & MU01_gar1_5f_for & GGTAACGCCAGGGTTTTCCCAGTCACGACGGTTTAAACTTATATCCACCGTGTCCCAG & 1610 \\
\hline & MU02_gar1_5f_rev & GACGCAGTTGTTTGAGCAAC & \\
\hline \multirow[t]{2}{*}{ trpC terminator (Aspergillus) } & MU03_trpCt_for & GTGGATAACCCCATCTTCAAGCAGTCCTGAGATCCACTTAACGTTACTGAAATCA & 803 \\
\hline & MU04_trpCt_rev & GAGTGGAGATGTGGAGTGGG & \\
\hline \multirow[t]{2}{*}{ gar1 3'direct repeat (tre22004) } & MU05_gar1_3dr_for & TGTGTAAGCGCCCACTCCACATCTCCACTCGGCGCGCCTTGCATTGGTCAGAGCGGTA & 361 \\
\hline & MU06_gar1_3dr_rev & CGAGAGCAGAGCAGCAGTAGTCGATGCTAGGCGGCCGCCCGACTTGGAGAAGCTCGTC & \\
\hline \multirow[t]{2}{*}{ garl 3'flank (tre22004) } & MU07_gar1_3f_for & CCAGCTGCGATTGATGTGTATCTTTGGATGGCGGCCGCTTGCATTGGTCAGAGCGGTA & 1576 \\
\hline & MU08_gar1_3f_rev & AGCGGATAACAATTTCACACAGGAAACAGCGTTTAAACAAGCAGTGGATGACTTGCTG & \\
\hline \multirow[t]{2}{*}{ cbh1 promoter (tre123989) } & MU09_cbh1p_for & AGGTTAGTAGGTTGCTCAAACAACTGCGTCGGCCGGCCTGTGGCAACAAGAGGCCAGA & 1646 \\
\hline & MU10_cbh1p_rev & GGCAGCGCCGGTGACGAGCAGGCGCTTCATGATGCGCAGTCCGCGGTTGA & \\
\hline \multirow[t]{2}{*}{ cDNA1 promoter (tre123515) } & MU11_cDNA1p_for & AGGTTAGTAGGTTGCTCAAACAACTGCGTCGGCCGGCCGAATTCGGTCTGAAGGACGT & 1231 \\
\hline & MU12_cDNA1p_rev & GGCAGCGCCGGTGACGAGCAGGCGCTTCATGTTGAGAGAAGTTGTTGGATTGA & \\
\hline \multirow[t]{2}{*}{ pdc1 promoter (tre121534) } & MU13_pdc1p_for & AGGTTAGTAGGTTGCTCAAACAACTGCGTCGGCCGGCCAAAGGAGGGAGCATTCTTCG & 1370 \\
\hline & MU14_pdc1p_rev & GGCAGCGCCGGTGACGAGCAGGCGCTTCATGATTGTGCTGTAGCTGCGCT & \\
\hline
\end{tabular}

The gene identifiers (tre-numbers) refer to Joint Genome Institute T. reesei assembly release version 2.0 
carbon sources, as indicated. Medium for 24-well plate cultivations contained $15 \mathrm{~g} \mathrm{~L}^{-1}$ lactose with $10 \mathrm{~g} \mathrm{~L}^{-1}$ D-galacturonate or $10 \mathrm{~g} \mathrm{~L}^{-1}$ lactose with $20 \mathrm{~g} \mathrm{~L}^{-1}$ pectin (Sigma P1935, Table 2). Immediately before inoculation, $1 \mathrm{~mL} \mathrm{~L}^{-1}$ Pectinex Ultra SP-L (Novozymes) was added to pectin-containing medium. All 24-well plate medium also contained $100 \mathrm{mM}$ PIPPS and $1 \mathrm{~g} \mathrm{~L}^{-1}$ spent grain extract, with the $\mathrm{pH}$ adjusted to 5.5 .

Pre-cultures for bioreactors were grown in low phosphate medium with $20 \mathrm{~g} \mathrm{~L}^{-1}$ lactose and $1 \mathrm{~g} \mathrm{~L}^{-1}$ yeast extract.

Media for bioreactors contained $15 \mathrm{~g} \mathrm{~L}^{-1}$ lactose and $7.5 \mathrm{~g} \mathrm{~L}^{-1}$ Sigma pectin or $9 \mathrm{~g} \mathrm{~L}^{-1}$ lactose and $11 \mathrm{~g} \mathrm{~L}^{-1}$ Meridianstar Rapid set pectin (Table 2) in the batch phase, plus $3 \mathrm{~g} \mathrm{~L}^{-1}$ yeast extract. Filter sterilised Pectinex Ultra SP-L $\left(1 \mathrm{~mL} \mathrm{~L}^{-1}\right)$ was added to the reactors after sterilisation at 1 and $10 \mathrm{~L}$ scale, but unsterilized enzyme was added before sterilisation for the $250 \mathrm{~L}$ pilot. Feed for the fed-batch cultivations contained $1 \mathrm{~g} \mathrm{~L}^{-1}$ yeast extract and either $50 \mathrm{~g} \mathrm{~L}^{-1}$ Sigma pectin with $6 \mathrm{~g} \mathrm{~L}^{-1}$ lactose or $78 \mathrm{~g} \mathrm{~L}^{-1}$ Meridianstar pectin. Pectin in the feed was hydrolysed by the addition of $1.0 \mathrm{~mL} \mathrm{~L}^{-1}$ Pectinex Ultra SP-L with $0.1 \mathrm{~mL} \mathrm{~L}^{-1}$ Pectinex Smash (Novozymes) and incubation at $\sim 45{ }^{\circ} \mathrm{C}$, with agitation, prior to sterilisation. Hydrolysis was allowed to proceed until the viscosity had been reduced to allow adequate sterilisation. The D-galacturonic acid concentration was assessed by HPLC and if hydrolysis was not yet complete, additional sterile Pectinex Ultra SP-L was added after sterilisation to ensure the availability of $\mathrm{D}$-galacturonic acid for the process.

\section{Culture conditions}

Twenty-four-well plates (Whatman $10 \mathrm{~mL}$ round bottom Uniplate) contained $4 \mathrm{~mL}$ medium per well, with either $10 \mathrm{~g} \mathrm{~L}^{-1}$ D-galacturonate or $20 \mathrm{~g} \mathrm{~L}^{-1}$ pectin (Sigma), which was enzymatically hydrolysed simultaneously with the cultivation, as the substrate and lactose as the carbon source for growth. Wells were inoculated with approximately $10^{5}$ spores in $10 \mu \mathrm{L}$ suspension $\left(\sim 2.5 \times 10^{4}\right.$ spores $\mathrm{mL}^{-1}$ final concentration). One well for each medium was not inoculated and used as a control. Three wells were inoculated with each new transformant (expressing $u d h$ under the CBH1, PDC1 and $c D N A 1$ promoters) and one well each with M122 (negative control) and VTT D-161646. The plates were covered with adhesive, breathable rayon fibre film for culture plates (VWR) and incubated at $28{ }^{\circ} \mathrm{C}, 800 \mathrm{rpm}$ (Infors HT Microtron, Switzerland), $85 \%$ relative humidity for up to 7 days. Samples $(100 \mu \mathrm{L})$ were taken daily after $72 \mathrm{~h}$ using wide-mouth $200 \mu \mathrm{L}$ pipette tips and either diluted and analysed immediately or stored at $-20{ }^{\circ} \mathrm{C}$. Sample size was minimised for the 24-well plates to ensure adequate culture volume for continued incubation.

Pre-cultures for bioreactor inocula were started in Erlenmeyer flasks (500-2000 mL containing 20\% volume medium) inoculated with $\sim 1 \times 10^{5}$ spores $\mathrm{mL}^{-1}$ (final concentration) to provide a $10 \% \mathrm{v} / \mathrm{v}$ inoculum for 1,10 or $32 \mathrm{~L}$ reactors. The $32 \mathrm{~L}$ reactor (New Brunswick $32 \mathrm{~L}$ BioFlo 510, Eppendorf) was used to provide inoculum for the $300 \mathrm{~L}$ reactor. Flasks were incubated at $30^{\circ} \mathrm{C}$ with $200 \mathrm{rpm}$ agitation for approximately $45 \mathrm{~h}$ before being transferred directly to bioreactors to provide an initial biomass of about $1 \mathrm{~g} \mathrm{~L}^{-1}$.

Bioreactor cultures were grown in Multifors (Infors HT, Switzerland, max. working volume $0.5 \mathrm{~L}$ ), Biostat Q (Sartorius AG, Germany, 1 L max. working volume), Biostat C (Sartorius AG, Germany, 10 L max. working volume), BioFlo 510 (Eppendorf) or New Brunswick Scientific IF400 (Eppendorf, $350 \mathrm{~L}$ max. working volume) bioreactors. Cultures were maintained at $35{ }^{\circ} \mathrm{C}$, with 500-900 rpm (0.5 L), 350-500 rpm (1 L), $400 \mathrm{rpm}$ $(10 \mathrm{~L}), 200 \mathrm{rpm}(32 \mathrm{~L})$ or $125-200 \mathrm{rpm}(350 \mathrm{~L}$ reactor $)$ agitation and approximately 0.5 volume gas (volume culture $)^{-1} \mathrm{~min}^{-1}(\mathrm{vvm})$. Culture $\mathrm{pH}$ was kept constant at $\mathrm{pH}$ 4.0 by the addition of sterile $2-4 \mathrm{M} \mathrm{NaOH}$ or $1 \mathrm{M} \mathrm{H}_{3} \mathrm{PO}_{4}$. Gas concentration $\left(\mathrm{CO}_{2}, \mathrm{O}_{2}, \mathrm{~N}_{2}\right.$ and Ar) was analysed continuously using a Prima Pro Process mass spectrometer (Thermo Scientific, UK) calibrated with $3 \% \mathrm{CO}_{2}$ in $\mathrm{Ar}, 5 \% \mathrm{CO}_{2}$ with $0.99 \%$ Ar and $15 \% \mathrm{O}_{2}$ in $\mathrm{N}_{2}, 20 \% \mathrm{O}_{2}$ plus $20 \% \mathrm{Ar}$ in $\mathrm{N}_{2}$, and $0.04 \%$ ethanol in $\mathrm{N}_{2}$. Fed-batch cultures were provided feed at a constant rate after at least $5 \mathrm{~g} \mathrm{~L}^{-1}$ biomass had been produced. The start of feeding for the $1 \mathrm{~L}$ culture was delayed to allow complete utilisation of the lactose, but this appeared to reduce productivity and the feed for the $250 \mathrm{~L}$ culture (using the same substrate) was started with about $2 \mathrm{~g} \mathrm{~L}^{-1}$ lactose still present in the medium, as was the $10 \mathrm{~L}$ culture.

\section{Chemical analyses}

The concentrations of D-glucose, lactose, D-galactose, D-galacturonic acid, and galactaric acid were determined by HPLC using a Fast Acid Analysis Column $(100 \times 7.8 \mathrm{~mm}$, BioRad Laboratories, Hercules, CA $)$ linked to an Aminex HPX-87H organic acid analysis column $(300 \times 7.8 \mathrm{~mm}$, BioRad Laboratories $)$ with $5 \mathrm{mM}$ $\mathrm{H}_{2} \mathrm{SO}_{4}$ as eluent and a flow rate of 0.3 or $0.5 \mathrm{~mL} \mathrm{~min}{ }^{-1}$. The column was maintained at $55{ }^{\circ} \mathrm{C}$. Peaks were detected using a Waters 410 differential refractometer and a Waters 2487 dual wavelength UV (210 nm) detector.

Samples were diluted with eluent to give expected concentrations of galactaric acid between 1 and $3 \mathrm{~g} \mathrm{~L}^{-1}$ and heated at $100{ }^{\circ} \mathrm{C}$ for $1 \mathrm{~h}$ to solubilise crystals of galactaric acid prior to HPLC analysis. Samples from 24-well plates 
were diluted without separation of biomass and supernatant, heated at $100{ }^{\circ} \mathrm{C}$ for $0.5-1 \mathrm{~h}$, then centrifuged at room temperature to remove biomass.

\section{Downstream processing}

Culture broth was collected from the 1,10 and $250 \mathrm{~L}$ cultures and the mycelium separated from the supernatant by filtration. At the 1 and $10 \mathrm{~L}$ scale, the mycelium was filtered through thick cleaning cloth under vacuum. The $250 \mathrm{~L}$ culture was warmed to $45^{\circ} \mathrm{C}$ before biomass was separated from the liquid using Seitz K300 depth filter sheets (Pall Corporation) in a Seitz 40-30A4 filter press to obtain approximately $250 \mathrm{~L}$ filtrate and $20 \mathrm{~kg}$ wet biomass. The supernatant was transferred during filtration to 2 large vessels (165 and $85 \mathrm{~L}$ ) which were cooled via cold jackets operated with water circulated from a chiller to give temperatures between 7 and $12{ }^{\circ} \mathrm{C}$. One and $10 \mathrm{~L}$ volumes were stored at $4{ }^{\circ} \mathrm{C} . \mathrm{HCl}$ was added to all culture supernatants to reduce the $\mathrm{pH}$ to between 1.5 and 2.2. Supernatants with precipitating galactaric acid crystals were mixed periodically by shaking ( 1 and $2 \times 5 \mathrm{~L}$ ) or stirring with a paddle (165 and $85 \mathrm{~L}$ ) until it was convenient to collect the crystals of galactaric acid.

Galactaric acid crystals were collected by filtration through Whatman GF/A filter paper. For large volumes, the crystals were first allowed to settle to the bottom of the container and the clear liquid decanted to a clean vessel. The decanted liquid was expected to contain some galactaric acid and was returned to the cold to allow further crystal formation, precipitation and collection. The process was repeated twice for the $1 \mathrm{~L}$ culture supernatant and four times for the 10 and $250 \mathrm{~L}$ volumes.

\section{Biomass determination}

Mycelia were collected by filtration through Whatman GF/B filters under vacuum and washed twice with an equal or greater volume of distilled $\mathrm{H}_{2} \mathrm{O}$. Mycelia were dried to a constant weight at $105^{\circ} \mathrm{C}$.

\section{Authors' contributions}

AH carried out all work related to scaling down the process (transformations and 24-well plate cultivations). TP carried out the scaling up cultivations. MW designed the study, supervised the scaling-up and carried out HPLC analysis and DSP. MW and AH drafted the manuscript. All authors read and approved the final manuscript.

\section{Acknowledgements}

We thank Merja Aarnio for technical assistance.

\section{Competing interests}

The authors declare that they have no competing interests.

\section{Availability of data and materials}

The datasets used and analysed during the current study are available from the corresponding author on reasonable request.
Funding

This research was supported by the VTT BioEconomy Programme.

\section{Publisher's Note}

Springer Nature remains neutral with regard to jurisdictional claims in published maps and institutional affiliations.

Received: 14 April 2017 Accepted: 6 July 2017

Published online: 11 July 2017

\section{References}

1. Boussie TR, Dias EL, Fresco ZM, Murphy VJ, Shoemaker J, Archer R, et al. Adipic acid composition. 2011; Patent WO2011/109051 A1.

2. Thomas D, Asikainen $M$, Harlin A. Method for producing muconic acids and furans from aldaric acids. 2015; Patent WO2015189481 A1.

3. Lavilla C, Alla A, Martínez de llarduya A, Benito E, García-Martín MG, Galbis JA, Muñoz-Guerra S. Carbohydrate-based polyesters made from bicyclic acetalized galactaric acid. Biomacromolecules. 2011;12:2642-52.

4. Kiely DE, Kirk RHS. Method of oxidation using nitric acid. 2010; Patent US7692041 B2.

5. Liu Y, Gong X, Wang C, Du G, Chen J, Kang Z. Production of glucaric acid from myo-inositol in engineered Pichia pastoris. Enzym Microb Technol. 2016;91:8-16

6. Zhang H, Li X, Su X, Ang EL, Zhang Y, Zhao H. Production of adipic acid from sugar beet residue by combined biological and chemical catalysis. ChemCatChem. 2016:8:1500-6.

7. Barth D, Wiebe MG. Enhancing fungal production of galactaric acid. Appl Microbiol Biotechnol. 2017;101:4033-40.

8. Mojzita D, Wiebe M, Hilditch S, Boer H, Penttilä M, Richard P. Metabolic engineering of fungal strains for conversion of D-galacturonate to mesogalactarate. Appl Environ Microbiol. 2010;76:169-75.

9. Wiebe MG, Mojzita D, Hilditch S, Ruohonen L, Penttilä M. Bioconversion of D-galacturonate to keto-deoxy-L-galactonate (3-deoxy-L-threo-hex2-ulosonate) using filamentous fungi. BMC Biotechnol. 2010;10:63.

10. Kuivanen J, Wang Y-MJ, Richard P. Engineering Aspergillus niger for galactaric acid production: elimination of galactaric acid catabolism by using RNA sequencing and CRISPR/Cas9. Microb Cell Fact. 2016;15:210.

11. Formenti LR, Nørregaard A, Bolic A, Hernandez DQ, Hagemann T, Heins AL, Larsson H, Mears L, Mauricio-Iglesias M, Krühne U, Gernaey KV. Challenges in industrial fermentation technology research. Biotechnol J. 2014;9:727-38.

12. Running JA, Bansal K. Oxygen transfer rates in shaken culture vessels from Fernbach flasks to microtiter plates. Biotechnol Bioeng. 2016;113:1729-35.

13. Linde T, Hansen NB, Lübeck M, Lübeck PS. Fermentation in 24-well plates is an efficient screening platform for filamentous fungi. Lett Appl Microbiol. 2014;59:224-30.

14. Dana CM, Dotson-Fagerstrom A, Roche CM, Kal SM, Chokhawala HA, Blanch HW, Clark DS. The importance of pyroglutamate in cellulase Cel7A Biotechnol Bioeng. 2014;111:842-7.

15. Landowski CP, Mustalahti E, Wahl R, Croute L, Sivasiddarthan D, Westerholm-Parvinen A, Sommer B, Ostermeier C, Helk B, Saarinen J, Saloheimo M. Enabling low cost biopharmaceuticals: high level interferon alpha-2b production in Trichoderma reesei. Microb Cell Fact. 2016;15:104.

16. Nguyen EV, Imanishi SY, Haapaniemi P, Yadav A, Saloheimo M, Corthals GL, Pakula TM. Quantitative site-specific phosphoproteomics of Trichoderma reesei signaling pathways upon induction of hydrolytic enzyme production. J Proteome Res. 2016;15:457-67.

17. Li J, Wang J, Wang S, Xing M, Yu S, Liu G. Achieving efficient protein expression in Trichoderma reesei by using strong constitutive promoters. Microb Cell Fact. 2012;11:84

18. Kuorelahti S, Kalkkinen N, Penttilä M, Londesborough J, Richard P. Identification in the mold Hypocrea jecorina of the first fungal D-galacturonic acid reductase. Biochemistry. 2005:44:11234-40.

19. Gietz RD, Woods RA. Transformation of yeast by lithium acetate/singlestranded carrier DNA/polyethylene glycol method. Guid Yeast Genet Mol Cell Biol Pt B. 2002;350:87-96. 
20. Korppoo A. Development of the CRISPR/Cas9 method for use in T. reesel. University of Helsinki; 2017. https://helda.helsinki.fi/bitstream/handle/10138/175220/developm.pdf?sequence=1. Accessed 19 Mar 2017.

21. Gruber F, Visser J, Kubicek CP, Degraaff LH. The development of a heterologous transformation system for the cellulolytic fungus Trichoderma reesei based on a pyrg-negative mutant strain. Curr Genet. 1990;18:71-6.
22. Penttilä M, Nevalainen H, Rättö M, Salminen E, Knowles J. A versatile transformation system for the cellulolytic filamentous fungus Trichoderma reesei. Gene. 1987;61:155-64.

\section{Submit your next manuscript to BioMed Central and we will help you at every step:}

- We accept pre-submission inquiries

- Our selector tool helps you to find the most relevant journal

- We provide round the clock customer support

- Convenient online submission

- Thorough peer review

- Inclusion in PubMed and all major indexing services

- Maximum visibility for your research

Submit your manuscript at

www.biomedcentral.com/submit 